Review Article

\title{
Focus on Diffusion MR Investigations of Musculoskeletal Tissue to Improve Osteoporosis Diagnosis: A Brief Practical Review
}

\author{
Silvia Capuani, ${ }^{1,2}$ Guglielmo Manenti, ${ }^{3}$ Riccardo Iundusi, ${ }^{2}$ and Umberto Tarantino ${ }^{2}$ \\ ${ }^{1}$ CNR-IPCF UOS Roma Sapienza, Physics Department, "Sapienza" University of Rome, Italy \\ ${ }^{2}$ Department of Orthopaedics and Traumatology, PTV Foundation, "Tor Vergata” University of Rome, Italy \\ ${ }^{3}$ Department of Diagnostic and Interventional Radiology, Molecular Imaging and Radiotherapy, PTV Foundation, \\ "Tor Vergata" University of Rome, Italy \\ Correspondence should be addressed to Silvia Capuani; silvia.capuani@romal.infn.it
}

Received 28 October 2014; Accepted 18 February 2015

Academic Editor: Jimin Ren

Copyright (C) 2015 Silvia Capuani et al. This is an open access article distributed under the Creative Commons Attribution License, which permits unrestricted use, distribution, and reproduction in any medium, provided the original work is properly cited.

\begin{abstract}
Nowadays, a huge number of papers have documented the ability of diffusion magnetic resonance imaging (D-MRI) to highlight normal and pathological conditions in a variety of cerebral, abdominal, and cardiovascular applications. To date, however, the role of D-MRI to investigate musculoskeletal tissue, specifically the cancellous bone, has not been extensively explored. In order to determine potentially useful applications of diffusion techniques in musculoskeletal investigation, D-MRI applications to detect osteoporosis disease were reviewed and further explained.
\end{abstract}

\section{Introduction}

Since the early publications by Le Bihan [1] and Basser [24 ] a great number of papers have documented the ability of diffusion magnetic resonance imaging (D-MRI) to define and discriminate normal and pathological conditions in a variety of cerebral $[5,6]$, abdominal $[7-10]$ and cardiovascular applications [11, 12]. The enormous importance of D-MRI techniques in clinical diagnostics is due to the fact that biological water in tissues is an endogenous molecular probe and its diffusion reflects tissue configuration at a microscopic level. Therefore, diffusion magnetic resonance (MR) images can measure water proton displacements, within and between tissues, by probing molecules motion on the micrometer length scale which is orders of magnitude smaller than the macroscopic MR image resolution (usually 1-3 millimeters, in clinical applications).

To date, however, the potential role of D-MRI in the diagnosis of musculoskeletal disorders has not been extensively explored [13]. This is due to different causes of various nature that we have focused and described here, on the base of our experience as interdisciplinary group consisting of academics researchers in physics, radiologist clinicians, orthopedic surgeons and clinicians operating in the field of musculoskeletal diseases.

In the past, technical restriction limited the use of DMRI techniques to the brain $[5,6,12]$. However, during the last 10 years, with the improvement in scanners technology and the availability of new MR sequences, investigation of the musculoskeletal system was made possible [14-23]. By using "water diffusion imaging" and "musculoskeletal" as key words for literature search, about twenty reviews are found. Some of these are focused on D-MRI for differentiation of acute benign and neoplastic vertebral compression fractures [14-16], painful spinal disorders [17], musculoskeletal tumor imaging $[18,19]$, and spine, muscles, and peripheral nerves disorders [20]. Some others are focused on D-MRI in body [21] and in pediatric radiology [22, 23]. However, none of these reviews is specifically focused on water diffusion MRI to detect osteoporosis. On the other hand, by using the following key words for literature search, "water diffusion imaging" and "osteoporosis," only one relevant review, 
published in 2010, is obtained [14]. In this review, only very few studies about vertebral marrow diffusion in osteoporosis are reported, which show contradictory results.

Recently, in parallel with technological development, a new point of view about the study of the bone marrow water in cancellous bone has been introduced. This new point of view provides an explanation of the previous contradictory results extending the water diffusion investigation to other skeletal sites in addition to the vertebral one.

From the above considerations, the need for this review, which includes and explains the previous and the last recent results on the basis of simple and intuitive considerations regarding the biophysical mechanism of water diffusion in the tissues. Recommendations and suggestions about practical steps of optimization closely related to the microstructural characteristics of the different skeletal sites are also reported.

Osteoporosis is a systemic and metabolic disorder characterized by a progressive reduction of mineral bone mass and microarchitectural deterioration of bone tissue, which increases the risk of bone fractures [24, 25]. The clinical diagnosis of osteoporosis is currently based on the quantification of bone mineral density (BMD) performed by dualenergy X-ray absorptiometry (DXA) [26]. Although DXA examination is economical and noninvasive, it is known to have reduced sensitivity and a low predictive value on patients' risk of reporting bone fracture $[27,28]$. This lack of sensitivity is likely to be due to the partial information that BMD provides on cancellous bone characteristics, assessing exclusively its mineral component [27, 29]. Indeed, bone strength depends on BMD and bone quality [25, 30, 31]. Bone quality refers to topological properties of trabecular microstructure, bone turnover, and composition of bone marrow. These and other factors, such as collagen framework and cell viability [32], may contribute to determining bone strength and its resistance to fracture [29]. In this regard, magnetic resonance MR techniques allow investigation of both trabecular networks and bone marrow [25, 33-37] providing some additional information on the physiological and functional changes associated with osteoporosis [29].

In general, D-MRI techniques are more challenging when applied to extracranial tissues due to technical issues mainly related to magnetic susceptibility variation $\Delta \chi$ and motion sensitivity [13]. In particular, water diffusion in bone marrow and cancellous bone is affected by strong internal magnetic field gradients $\left(G_{i}\right)$ generated by $\Delta \chi[38,39]$ and mainly localized at the interface between bone and water components [40].

The setting, optimization, and operation of D-MRI techniques in cancellous bone and muscle investigations are markedly different from conventional MRI of the musculoskeletal system primarily based on image contrast due to differences in tissues relaxation times: $T_{1}, T_{2}$, and $T_{2}^{*}$ [41, 42]. Therefore, D-MRI applications demand a greater level of knowledge of the diffusion fundamentals, attention, and forethought. Currently, thanks to the development of high performance magnetic field gradients, new diffusion sensitized sequences, and parallel imaging techniques, the main technical problems are drastically reduced. Moreover, a new point of view on human cancellous bone $[40,43]$, described as a porous system in which water experiences a regime of restricted diffusion in the space between fat and mineral bone in each pore, forces to develop new MRI protocols based on molecular diffusion of water to obtain new microstructural information for improving osteoporosis diagnosis.

The basic principles of diffusion and its assessment of D-MRI in musculoskeletal tissue were summarized here, together with a brief description about microstructural characteristics of musculoskeletal tissues. Subsequently, some strategies and suggestions to optimize D-MRI protocols for their potential applications in the diagnosis of osteoporosis were described.

\section{Microstructural Characteristics of Musculoskeletal Tissues}

The principal musculoskeletal tissues that are related to osteoporosis and can be investigated with D-MRI are bones [33-38], bone marrow, muscles, and fat [44-48].

Bone tissue is a complex biomaterial composed of a solid mineral matrix, which is filled by bone marrow (a soft interstitial material). The solid matrix is mainly constituted by mineral components while bone marrow is mainly composed by water and fat at different relative percentages. The relative concentration of each bone marrow component is dependent on both its anatomical skeletal location and the age of considered individuals [49]. Human bone may be classified as cortical or trabecular. The former is mainly present in the shaft of long bones and is much denser (with a porosity ranging from $5 \%$ to $10 \%$ ) than cancellous bone. Conversely, cancellous bone is much more porous (with a porosity ranging from 50\% to $90 \%$ ), and it is metabolically more active. From a physical point of view, cancellous bone is a porous system that can be described as a solid with holes and cavities, that is, presenting connected void spaces, randomly distributed within a solid matrix. Moreover, water is more prevalent in the boundary zone while fat occupies primarily the central zone of each pore, as recently demonstrated [40]. Water in the boundary zones of pores is also due to the presence of the endosteum, a thin membrane (with size about 3$7 \mu \mathrm{m}$ ) of soft tissue (principally water) comprised of a linear chain of cells that lines the medullary cavity [50]. Moreover, due to a biological division of the bone marrow compartment, granulocytes and other nonfat entities accumulate at the boundary of the bone marrow compartment adjacent to the endosteum. Pore sizes in human cortical bone are in the range of several nanometers to few micrometers, while pore sizes in cancellous bone are in the range $1 \mu \mathrm{m}-1 \mathrm{~mm}$, where intertrabecular plates space ranges approximately from $100 \mu \mathrm{m}$ to $1-2 \mathrm{~mm}$. On the other hand, the space between fat and bone in the boundary of each pore ranges from less than $1 \mu \mathrm{m}$ (when endosteum is damaged or absent due to pathologies) to $10-20 \mu \mathrm{m}$ [50] or more, in the case of trabecular disruption or bone marrow irradiation. Bone marrow located inside the central cavities of long bones is not forced in pores. From a microstructural point of view, both bone marrow in trabecular bone and bone marrow free in the larger bone cavities are soft tissues characterized by several particles of spherical/elliptical shape and average size ranging 
from 6 microns of red blood cells to approximately 100 microns of the fat globules. Adipose tissue is characterized by approximately the same kind of bone marrow fat [51]. Skeletal muscles are attached to and bring about the movement of the various bones of the skeleton. The whole muscle is enclosed in a sheath of connective tissue that folds inwards into the substance of the muscle to surround a large number of smaller bundles, called fasciculi. These fasciculi consist of still smaller bundles of elongated, cylindrical cells named fibres that are characterized by diameter size of about 10-20 $\mu \mathrm{m}$.

With the development of osteoporosis, a change of the aforementioned microstructures size occurs. Bone microarchitecture is rearranged or disrupted (such as conversion of plates to rods and loss of connectivity) [41, 52, 53] and bone marrow which fills the trabecular holes is altered [44$46,54]$. Specifically, an increase of the marrow fat that decreases the interstitial space size in which water diffuse and a microstructural deterioration with a pore enlargement that increases the interstitial space size and their interconnection occur [43]. These changes do not result equally along all skeletal sites. As an example, in calcaneus, with the onset of osteoporosis, no bone marrow increase is detected but only a trabecular bone deterioration is observed [43, 55]. Moreover, recent investigations show a correlation between the cancellous bone deterioration and the loss of muscle mass with a change of fibres diameter size, referred to as sarcopenia $[47,48]$.

\section{Magnetic Resonance Diffusion: Introduction and Concepts}

Diffusion is the process by which matter is transported from one part to another as a result of random molecular motion, also called Brownian motion [56]. This motion results from the thermal energy carried by diffusing molecules and it is characterized by a diffusion coefficient $D$ that provides information about the behavior of the molecular motion which in turn depends on the characteristics of the medium in which the diffusion occurs. Therefore, $D$ measurement of water molecules in human tissues, as a consequence of the interactions between molecules and obstacles that hinder and or restrict their motion, gives information about size, orientation, and shape of cellular structures $[1,3]$.

Currently, D-MRI is the only means able to measure and monitor the diffusion coefficient in vivo, with a completely noninvasive modality and without requiring exogenous contrast agents. MRI measurements of diffusion indirectly measure the displacements of diffusing molecules in one dimension.

In Gaussian diffusion approximation, the mean square displacement (MSD) of diffusing molecules is linearly proportional to the diffusion coefficient $D$ and the time $t$ during which the diffusion process is observed [56]:

$$
\mathrm{MSD}=2 \mathrm{ND} t
$$

where $N$ is the "dimensionality" of the space over which diffusion distances are measured.
By using relation (1), it is possible to evaluate the length scale $l$ or the characteristic length probed by diffusing molecules:

$$
l=\mathrm{MSD}^{1 / 2}
$$

By using relation (1) and (2) at human body temperature, during typical diffusion times of about $t=50 \mathrm{~ms}$, random water molecules move in a homogeneous and isotropic medium over distances around $l=30 \mu \mathrm{m}$ in the tridimensional case (i.e., $N=3$ ) and around $l=15 \mu \mathrm{m}$ along a chosen spatial direction (i.e., $N=1$ ). However, this is the case of free diffusion of bulk water, for which there are no barriers to molecules motion and $D \approx 3 * 10^{-3} \mathrm{~mm}^{2} / \mathrm{s}$.

Because most human cells and subcellular structures have smaller dimensions than those listed above, it is highly likely that water molecules will have many interactions with cellular components over such $t$ measurement interval [3]. As a consequence, water movement in tissues is neither entirely free nor random, being modified by interactions with hydrophobic lipid-containing cell membranes, intracellular organelles, and macromolecules and by flows within tubular channels such as blood vessels, capillaries, interconnected pores, and ducts. Water motion in tissue is therefore related to its microscopic and submicroscopic biological structure. As depicted in Figure 1, by using relation (1) and (2) and measuring $D$, it is possible to detect different water diffusion behavior, such as free, hindered, and restricted diffusion, in structures characterized by different size, and, more importantly, it is possible to estimate microstructures size. Specifically, if water diffusion is limited by a container (such as water in cortical bone pores, or intracellular water), when the molecules reach the boundaries, they are reflected and the MSD results confined in the container [56]. As a consequence, both $D$ and $l$ are lower than those of free bulk water.

\section{Magnetic Resonance Diffusion Measurement: DWI and ADC}

The molecular diffusion is uniquely assessed by diffusion weighted imaging (DWI) techniques $[1,3]$. DWI techniques are able to measure the water diffusivity by the application of a couple of diffusion sensitizing gradients (motion probing gradients) to a simple $T_{2}$-weighted spin-echo sequence, named pulse field gradient spin-echo (PFGSE) [57]. Signal losses on DWI are proportional to both diffusion coefficient of water molecules and the diffusion gradient strength used, as described by the signal attenuation $S(b)$ of DWI, given by

$$
S(b)=S(0) \exp (-b D),
$$

where $S(0)$ is the signal amplitude in absence of diffusing gradients and $\mathrm{b}$ is the so-called $b$-value that indicates the strength and duration of application of diffusion sensitizing gradients together with the diffusion time $t$ during which the diffusion phenomenon is observed:

$$
b=(\gamma \delta g)^{2}\left(\Delta-\frac{\delta}{3}\right)
$$




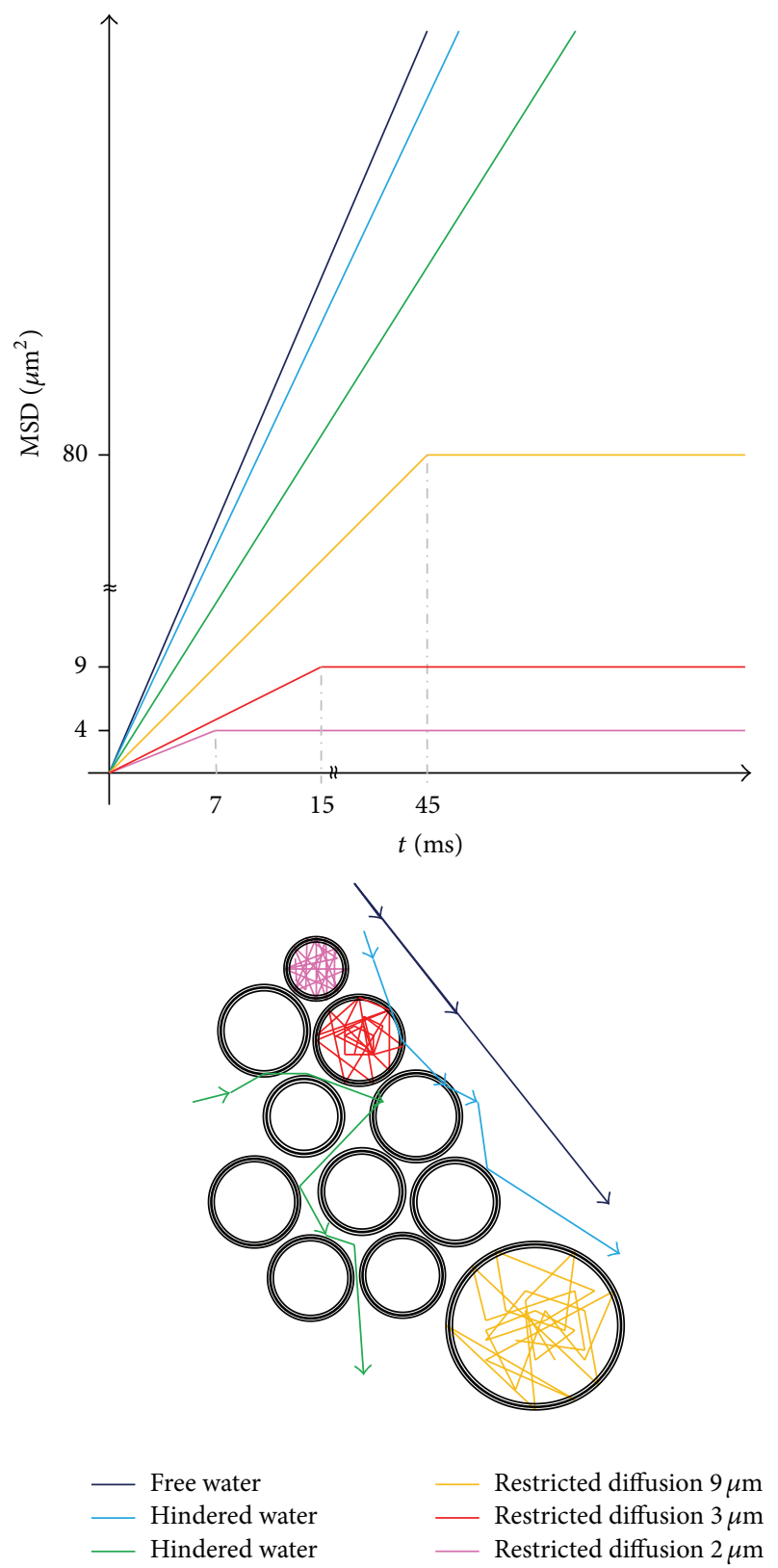

FIgURE 1: Schematic representation of water MSD as a function of the diffusion time $t$, obtained in a heterogeneous system depicted at the bottom. Solid lines represent curves obtained by using relation (1). In the case of restricted diffusion, the two solid lines represent curves obtained considering data at short and long times.

where $\gamma$ is the gyromagnetic ratio, $g$ is the strength of the gradient (diffusion gradient) used to encode diffusion, $\delta$ is the duration of the gradient pulse, and $\Delta$, which is the time between the leading edges of the pair of diffusion gradient pulses in PFGSE, is the diffusion time $t$ of relation (1). In the practice, the $b$-value which determines the degree of signal attenuation due to diffusion allows the selection of different diffusion behaviors characterized by different diffusion coefficients of water diffusing in heterogeneous systems (this issue will be discussed in paragraph 7).
By observing Figure 1, it is possible to understand that if the diffusion process is observed for time $t$ which is too short, the diffusing water molecules do not reach obstacles and walls of pore; in this case, a free diffusion behavior and a free diffusion coefficient are detected. Therefore, an adequate diffusion time must be selected to obtain microstructural information from DWI investigations. As an example, to detect the presence of pores characterized by pore size of about $9 \mu \mathrm{m}, t$ greater than $45 \mathrm{~ms}$ must be used with $D \approx$ $3 * 10^{-4} \mathrm{~mm}^{2} / \mathrm{s}$, as shown in Figure 1 . Moreover, free diffusion of water at human body temperature shows the highest $D$ value approximately equal to $3 * 10^{-3} \mathrm{~mm}^{2} / \mathrm{s}$. Hindered and restricted water diffusion assume smaller values with increasing of obstacles and with the decreasing of pores and structures size within which the water is confined.

Because in anisotropic and heterogeneous systems diffusion coefficient depends on both the direction along which the diffusion gradient acts and the diffusion time $t=\Delta$, DWI in tissues refers to $\mathrm{ADC}$, the apparent diffusion coefficient, rather than $D$, the self-diffusion constant of a pure isotropic and homogeneous solution. By using relation (3), the ADC can be computed in each voxel of DW image to obtain an ADC map according to the following:

$$
\mathrm{ADC}=-\left(\frac{1}{b}\right) \ln \left[\frac{S(b)}{S(0)}\right] .
$$

\section{Diffusion Tensor Imaging: DTI}

Molecular mobility in tissues which are in general heterogeneous and anisotropic is not the same in all directions [2-4]. As an example, water diffusion along muscles fibers is less hindered than that along directions perpendicular to fibers [58]. In the presence of anisotropy, diffusion can no longer be completely characterized by a single scalar coefficient but required a tensor, $\underline{D}$, which fully describes molecular mobility along each direction and correlation between these directions. Because $\underline{D}$ is symmetric, only six ADC measurements along six independent directions are needed, and relation (3) is now expressed by the following [2]:

$$
\begin{aligned}
S(\underline{D})=S(0) \exp ( & -b_{x x} D_{x x}-b_{y y} D_{y y}-b_{z z} D_{z z} \\
& \left.-2 b_{x y} D_{x y}-2 b_{x z} D_{x z}-2 b_{y z} D_{y z}\right),
\end{aligned}
$$

where, the on-diagonal terms $D_{x x}, D_{y y}$, and $D_{z z}$ represent the ADC along axes $x, y$, and $z$, respectively, while the offdiagonal terms $D_{x y}, D_{x z}$, and $D_{y z}$ reflect correlation between molecular displacements in perpendicular directions [2-4].

In practice, to determine $\underline{D}$, one must first collect DW images along at least six independent directions, by using diffusion sensitized MRI pulse sequences with diffusion gradients along six independent directions, such as $x, y, z$, $x y, x z$, and $z y$, plus a spin-echo image that provides the $S(0)$ term in relation (6). Therefore, DTI protocol lasts six times more than the DWI protocol; however, from the tensor, several pieces of information can be extracted. The sun of the on-diagonal elements is rotationally invariant measure of diffusion, that is, a measure of diffusion independent on the 
reference frame. This is very important in diagnostic applications because diffusion quantification obtained at different times in a given patient or in different patients and healthy subjects or in different hospitals can be compared. One-third of the trace is often referred to as the mean diffusivity (MD) and constitutes a directionally averaged diffusion coefficient that can also be obtained by averaging the ADC measured along $x, y$, and $z$ directions. The fractional anisotropy (FA), a sort of normalized standard deviation, measures the fraction of the magnitude of $\underline{D}$ that can be ascribed to anisotropic diffusion. FA varies between 0 (isotropic diffusion) and 1 (maximum anisotropy). As an example, biceps femoris short head in a healthy subject is characterized by FA $=0.4$ [59], but the same tissue in the presence of edema shows FA values less than 0.3 .

\section{D-MRI Pulses Sequences}

Diffusion gradients can be applied to many standard MRI sequences, but, particularly in musculoskeletal applications, the images produced are prone to artifacts resulting from physiological motions, eddy currents, and internal gradients due to $\Delta \chi$. Spin-echo DWI sequences such as pulse gradient spin-echo (PGSE) [57] or pulse gradient stimulated echo (PGSTE) [60] that is particularly useful to investigate diffusion in tissues characterized by $T_{2} \ll T_{1}$ (such as the most musculoskeletal tissues) yield high signal-to-noise ratio (SNR) images and are more resistant to field inhomogeneity created by the application of diffusion gradients. However, long acquisition times of several minutes limit their clinical use due to the increased likelihood of encountering motionrelated artifacts.

Because the reliability of diffusion contrast depends on SNR of DWI images [61], it must be approximately higher than 2 [62] to exclude an underestimation of the MD and an overestimation of the anisotropy quantified by FA $[62,63]$.

Single shot echo planar imaging (SS-EPI) sequences offer significantly faster acquisition times, thus overcoming DWI sensitivity to patient movement while still offering a relatively high SNR.

A major limitation of diffusion sensitized SS-EPI imaging is magnetic susceptibility artifacts, at different tissue interfaces common in musculoskeletal imaging, such as bone and soft tissue, which can lead to severe geometric image distortion, relatively low spatial resolution due to rapid $T_{2}^{*}$ decay of signal, and in general low image quality.

Recent advances in SS-EPI to overcome these problems include improved gradient systems with reduced eddy current effects which can help reduce geometric distortions and parallel imaging techniques employing multiple receiver coil elements to improve acquisition times and increase spatial resolution.

An alternative approach is to acquire EPI data using a segmented or multishot, echo planar readout (MS-EPI), which divides the echo train into several shorter parts. This makes the image less sensitive to susceptibility artifacts, reduces image distortion, and increases spatial resolution but at the price of longer acquisition times and therefore at a greater risk of motion artifact.
Recently [64], by using a diffusion sensitized segmented EPI with EPI factor equal to 7 and a scan time duration for the DTI protocol equal to 17:50 minutes, we have obtained the first MD and FA maps of femoral neck performed in three different groups of women based on their BMD (healthy, osteopenic, and osteoporotic women) [64]. Each DW image, acquired by using four averaged scans, was characterized by a sufficient SNR to extract significant values of MD and FA and an in-plane resolution of about $2.2 \mathrm{~mm}$ with a slice thickness of $5 \mathrm{~mm}$ [64]. Subsequently, the same diffusion sensitized segmented EPI sequence has been used to evaluate ADC in calcanei of healthy and osteoporotic subjects $[43,55]$.

\section{D-MRI Parameters for Optimization}

The choice of pulse sequence parameters has a profound influence on the accuracy and precision in the estimates $\mathrm{ADC}, \mathrm{MD}$, and FA and other diffusion metrics parameters. Currently, the most of these sequence parameters are already preset in clinical scanner to perform brain, spinal cord, or muscles investigations. For example, it is well known that DTI protocol with $b=1000 \mathrm{~s} / \mathrm{mm}^{2}$ is used in conventional neurological clinical applications $[6,7,12]$, while $b=500$ $800 \mathrm{~s} / \mathrm{mm}^{2}$ optimizes diffusion measurement in muscles [6567] and spinal cord [68]. Conversely, no suggestions can be usually found to perform diffusion investigation in cancellous bone.

In principle, the optimal $b$-value is approximately equal to the reciprocal of $\mathrm{ADC}$ (or $\mathrm{MD}$ ) that one is trying to estimate:

$$
b \approx \frac{1}{\mathrm{ADC}} .
$$

However, the best compromise between a sufficient SNR and a sufficient diffusion weighted contrast to detect diffusion of bone marrow water restricted between fat and bone must be reached.

Bone marrow fat extracted from the vertebrae, the femoral neck, and the calcaneus of healthy postmenopausal women was found to range from $50 \%$ to $70 \%, 60 \%$ to $88 \%$, and $78 \%$ to $98 \%$, respectively $[40,44,45,55,64,69-71]$. An increase in the amount of bone marrow fat involves a decrease of the interstitial space between bone and fat (region in which water diffuses), leading to a lower water ADC. As a consequence, water $\mathrm{ADC}$ is higher in the vertebrae, intermediate in femoral neck, and lower in the calcaneus; that is, diffusion of water in the calcaneus is more restricted compared to water in femoral neck or in vertebrae, as displayed in Figure 2.

Therefore, these three different skeletal sites must be probed with different length scale (because they are characterized by different pores size) and different $b$-values, because they are characterized by different water ADC. Specifically, ADC values of bone marrow water measured in human cancellous bone of healthy subjects showed the following approximate values: $\mathrm{ADC} \approx 10^{-3} \mathrm{~mm}^{2} / \mathrm{s}, \mathrm{ADC} \approx 4 *$ $10^{-4} \mathrm{~mm}^{2} / \mathrm{s}$, and ADC $\approx 5 * 10^{-5} \mathrm{~mm}^{2} / \mathrm{s}$ for the vertebrae, the femoral neck, and the calcaneus, respectively $[40,43,55,64$, $70,72,73]$. In principal, by using relation $(7), b$-values around $b=1000 \mathrm{~s} / \mathrm{mm}^{2}, b=2500 \mathrm{~s} / \mathrm{mm}^{2}$, and $b=20000 \mathrm{~s} / \mathrm{mm}^{2}$ 
Healthy cancellous bone

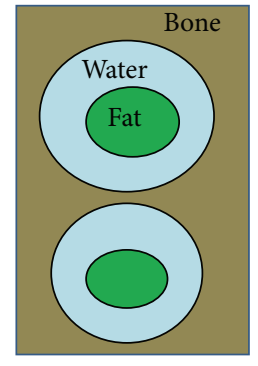

Vertebrae $\mathrm{ADC} \approx 10^{-3} \mathrm{~mm}^{2} / \mathrm{s}$

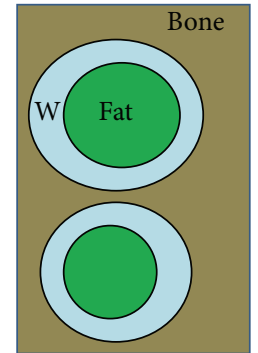

Femoral neck

$\mathrm{ADC} \approx 10^{-4} \mathrm{~mm}^{2} / \mathrm{s}$

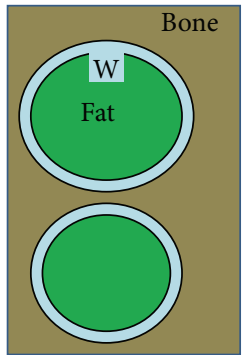

Calcaneus $\mathrm{ADC} \approx 10^{-5} \mathrm{~mm}^{2} / \mathrm{s}$

Osteoporotic cancellous bone
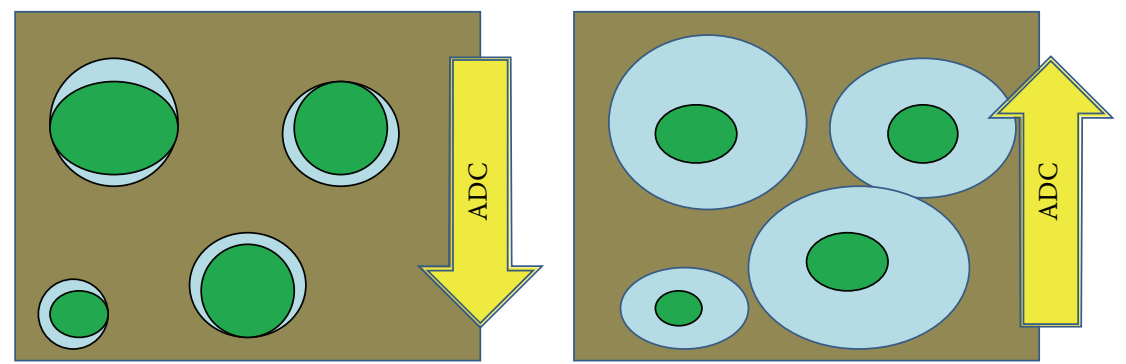

FIGURE 2: Schematic representation of cancellous bone at different skeletal sites in healthy (top) and osteoporotic (bottom) cancellous bone. Fat content increases from axial skeleton to peripheral skeletal sites. As a consequence, water ADC is higher in the vertebrae and lower in the calcaneus. Indeed, diffusion of water in calcaneus is more restricted compared to water in femoral neck or in vertebrae. With the development of osteoporosis, two different and opposite mechanisms occur: an increase of the marrow fat which decreases the ADC value and a microstructure deterioration with a pore enlargement that increases ADC. In the calcaneus, only the last mechanism results (adapted from reference [43] with permission).

should be used to measure ADC in vertebrae, femoral neck, and calcaneus, respectively. In reality, most tissues are made of multiple subcompartments (for example, the intra- and extracellular water compartments); each of these is characterized by a peculiar ADC. As a consequence, the ADC that is measured could depend on the range used for the $b$-values, as measurements with low $b$-values would be more sensitive to fast (near free or less hindered) diffusion components and measurements with high $b$-values would be more sensitive to slow (more restricted and entrapped) diffusion components. In the ideal case, a very large range of $b$ values would be required. However, this means long acquisition times incompatible with clinical applications. In any case, it is clear that it is better to estimate the averaged ADC in a tissue by using a range of $b$-values (from four to eight) and a fitting procedure of experimental data to relation (3) rather than estimate it using a single $b$-value.

As previously underlined, in cancellous bone, more than in other tissues, the magnetic susceptibility mismatch between the solid matrix and interstitial water bone marrow generates internal gradients of magnitude depending on the geometry and orientation of the trabeculae with respect to the static magnetic field direction scaling with magnetic field strength. Therefore, the $G_{i}$ strength that water molecules sense as they diffuse near the interface between fatty marrow and bone increases with the decreasing of the interstitial space and with the increasing of the static magnetic field
$[40,55,74]$. In particular, when cancellous bone in calcaneus is investigated, a positive coupling between $G_{i}$ and diffusion gradients occurs that produce an effective $b$-value higher than that computed by using relation (4). Finally, it is well known that the diffusive motion of water molecules through the $G_{i}$ leads to irreversible dephasing $[40,55,74-77]$. As a consequence, the effective diffusion coefficient $\mathrm{ACD}_{\text {eff }}$ (measured by using a DWI protocol) results in smaller than the ideal ADC obtained without taking into account the $\Delta \chi$ at the interfaces between water and bone [55, 78, 79]. Because of the above considerations, particularly in calcaneus D-MRI investigations, water $\mathrm{ADC}$ is underestimated and the use of a $b$-value range up to approximately $10000 \mathrm{~s} / \mathrm{mm}^{2}$ should be appropriate.

By considering relation (3) and (4), in order to achieve significant signal attenuation when the diffusion gradients are applied, the pulses must be strong. However, this places strong demands on the magnetic field gradient hardware. Therefore, D-MRI investigations in cancellous bone required high gradient unit performance of MRI scanner. The choice to select diffusion gradients applied for a long time (i.e., long $\delta$ ) to increase diffusion gradients strength is not applicable in cancellous bone and muscles investigations. Indeed, water in bone marrow and muscles is characterized by lower $T_{2}$, compared to other tissues (such as brain tissue). Therefore, long $\delta$ can lead to extended echo times, which consequently determines poor SNR in the resulting DW images. Moreover, 
due to the reduced $T_{2}$ of water in musculoskeletal tissues, the use of PGSTE (which requires $\Delta<T_{1}$ ) rather than PGSE (which requires $\Delta<T_{2}$ ) sequences would be preferred.

The $b$-value includes both diffusion time $\Delta$ and diffusion gradients strength $g$. Therefore, one can obtain certain $b$ value ranges by fixing the diffusion time $\Delta$ and varying $g$ or by fixing $g$ and varying $\Delta$. These two modalities do not provide equal investigations and equal results. This is because $\Delta$ also defines the length scale through which a tissue is probed while $g$ only determines the degree of signal attenuation due to diffusion.

Recently [43, 55], ADC values of bone marrow water measured in calcaneus showed an increasing trend from healthy $\left(\mathrm{ADC} \approx 4 * 10^{-11} \mathrm{~mm}^{2} / \mathrm{s}\right.$ ) to osteoporotic subjects (ADC $\left.\approx 7 * 10^{-11} \mathrm{~mm}^{2} / \mathrm{s}\right)$ and a significant negative correlation between $\mathrm{ADC}$ and $\mathrm{BMD}$ values. This study, performed with $b=8000 \mathrm{~s} / \mathrm{mm}^{2}$ and $\Delta=30 \mathrm{~ms}$, suggests that by using $l \approx 3 \mu \mathrm{m}$ healthy, osteopenic, and osteoporotic subjects can be significantly discriminated. This is most likely due to the fact that the spaces sizes between fat and bone (where the water diffuses) are around $3 \mu \mathrm{m}$ and they increase with the development of osteoporosis, according to the ADC increase $[43,55]$. On the other hand, healthy and osteoporotic subjects were significantly discriminated by selecting $b=2500 \mathrm{~s} / \mathrm{mm}^{2}$ and $\Delta=40 \mathrm{~ms}$, to probe femoral neck with $l \approx 10 \mu \mathrm{m}$ [64]. In this DTI study, both MD and FA values obtained in femoral neck showed a decreasing trend from healthy to osteoporotic subjects. These results suggest that, with the development of the osteoporosis, the space between fat and bone probed by water decreases due to marrow fat increases and it becomes more isotropic due to the structural rearrangement of the endosteum cell lines or of the trabeculae surface [64].

\section{Conclusion}

D-MRI in cancellous bone provides a new tool to probe tissue microstructures changes that occur with the development of osteoporosis. This is because at microscopic level many tissue features related to osteoporosis influence MR diffusion measurements.

Currently, diffusion in musculoskeletal tissue is quite difficult to use successfully, principally because of the occurrence of strong internal gradients due to magnetic susceptibility mismatch between soft tissue and mineral bone and short $T_{2}$. These obstacles, however, can sometimes be overcome with ad hoc optimized pulse sequences, hardware, and a theoretical background of diffusion process.

\section{Abbreviations by Order of Appearance in the Paper}

MR: Magnetic resonance

MRI: Magnetic resonance imaging

D-MRI: Diffusion magnetic resonance imaging

BMD: Bone mineral density

DXA: Dual-energy X-ray absorption

$\Delta \chi: \quad$ Magnetic susceptibility variation

$G_{i}: \quad$ Internal magnetic field gradients

MSD: Mean square displacement
D: $\quad$ Diffusion coefficient

$l$ : $\quad$ Length scale

DWI: Diffusion weighted imaging

DW: Diffusion weighted

PFGSE: Pulse field gradient spin-echo

$b$ : $\quad b$-value

$\gamma: \quad$ Gyromagnetic ratio

$g: \quad$ Strength of the magnetic field gradient

$\delta$ : $\quad$ Duration of the magnetic field gradient pulse

$\Delta: \quad$ Diffusion time: the time between the pair of diffusion gradient pulses

ADC: Apparent diffusion coefficient

DTI: Diffusion tensor imaging

$\underline{D}: \quad$ Diffusion tensor

MD: $\quad$ Mean diffusivity

FA: $\quad$ Fractional anisotropy

PGSE: Pulse gradient spin-echo

PGSTE: Pulse gradient stimulated echo

$T_{2}$ : $\quad$ Transverse relaxation time

$T_{1}$ : Longitudinal relaxation time

SNR: Signal-to-noise ratio

EPI: $\quad$ Echo planar imaging

SS-EPI: Single shot echo planar imaging

MS-EPI: Multishot echo planar imaging.

\section{Conflict of Interests}

The authors declare that there is no conflict of interests regarding the publication of this paper.

\section{Acknowledgment}

This work was supported by Agenzia Spaziale Italiana (Asi) "Studio multidisciplinare degli effetti della microgravità sulle cellule ossee" (SMEMCO). Call for research n. DC-DTE-2011033.

\section{References}

[1] D. Le Bihan, "Molecular diffusion nuclear magnetic resonance imaging," Magnetic Resonance Quarterly, vol. 7, no. 1, pp. 1-30, 1991.

[2] P. J. Basser, J. Mattiello, and D. LeBihan, "MR diffusion tensor spectroscopy and imaging," Biophysical Journal, vol. 66, no. 1, pp. 259-267, 1994.

[3] P. J. Basser, "Inferring microstructural features and the physiological state of tissues from diffusion-weighted images," NMR in Biomedicine, vol. 8, no. 7, pp. 333-344, 1995.

[4] P. J. Basser and C. Pierpaoli, "Microstructural and physiological features of tissues elucidated by quantitative-diffusion-tensor MRI," Journal of Magnetic Resonance Series B, vol. 111, no. 3, pp. 209-219, 1996.

[5] D. Le Bihan, R. Turner, P. Douek, and N. Patronas, "Diffusion MR imaging: clinical applications," The American Journal of Roentgenology, vol. 159, no. 3, pp. 591-599, 1992.

[6] M. A. Horsfield and D. K. Jones, "Applications of diffusionweighted and diffusion tensor MRI to white matter diseases-a review," NMR in Biomedicine, vol. 15, no. 7-8, pp. 570-577, 2002. 
[7] D.-M. Koh and D. J. Collins, "Diffusion-weighted MRI in the body: applications and challenges in oncology," American Journal of Roentgenology, vol. 188, no. 6, pp. 1622-1635, 2007.

[8] M. A. Haider, T. H. van der Kwast, J. Tanguay et al., "Combined T2-weighted and diffusion-weighted MRI for localization of prostate cancer," American Journal of Roentgenology, vol. 189, no. 2, pp. 323-328, 2007.

[9] M. P. Lichy, P. Aschoff, C. Plathow et al., "Tumor detection by diffusion-weighted MRI and ADC-mapping-initial clinical experiences in comparison to PET-CT,' Investigative Radiology, vol. 42, no. 9, pp. 605-613, 2007.

[10] S. Gourtsoyianni, N. Papanikolaou, S. Yarmenitis, T. Maris, A. Karantanas, and N. Gourtsoyiannis, "Respiratory gated diffusion-weighted imaging of the liver: value of apparent diffusion coefficient measurements in the differentiation between most commonly encountered benign and malignant focal liver lesions," European Radiology, vol. 18, no. 3, pp. 486-492, 2008.

[11] M.-T. Wu, W.-Y. I. Tseng, M.-Y. M. Su et al., "Diffusion tensor magnetic resonance imaging mapping the fiber architecture remodeling in human myocardium after infarction: correlation with viability and wall motion," Circulation, vol. 114, no. 10, pp. 1036-1045, 2006.

[12] H. P. Adams Jr., G. Del Zoppo, M. J. Alberts et al., "Guidelines for the early management of adults with ischemic stroke: a guideline from the American heart association/American stroke association stroke council, clinical cardiology council, cardiovascular radiology and intervention council, and the atherosclerotic peripheral vascular disease and quality of care outcomes in research interdisciplinary working groups," Stroke, vol. 38, no. 5, pp. 1655-1711, 2007.

[13] H. C. Thoeny and F. Keyzer, "Extracranial applications of diffusion-weighted magnetic resonance imaging," European Radiology, vol. 17, no. 6, pp. 1385-1393, 2007.

[14] A. Biffar, O. Dietrich, S. Sourbron, H.-R. Duerr, M. F. Reiser, and A. Baur-Melnyk, "Diffusion and perfusion imaging of bone marrow," European Journal of Radiology, vol. 76, no. 3, pp. 323$328,2010$.

[15] M. M. Y. Khoo, P. A. Tyler, A. Saifuddin, and A. R. Padhani, "Diffusion-weighted imaging (DWI) in musculoskeletal MRI: a critical review," Skeletal Radiology, vol. 40, no. 6, pp. 665-681, 2011.

[16] S. Colagrande, S. F. Carbone, L. M. Carusi, M. Cova, and N. Villari, "Magnetic resonance diffusion-weighted imaging: extraneurological applications," Radiologia Medica, vol. 111, no. 3, pp. 392-419, 2006.

[17] P. F. Beattie, "Diffusion-weighted magnetic resonance imaging of the musculoskeletal system: an emerging technology with potential to impact clinical decision making," Journal of Orthopaedic and Sports Physical Therapy, vol. 41, no. 11, pp. 887895, 2011.

[18] O. Dietrich, A. Biffar, M. F. Reiser, and A. Baur-Melnyk, "Diffusion-weighted imaging of bone marrow," Seminars in Musculoskeletal Radiology, vol. 13, no. 2, pp. 134-144, 2009.

[19] T. A. Bley, O. Wieben, and M. Uhl, "Diffusion-weighted MR imaging in musculoskeletal radiology: applications in trauma, tumors, and inflammation," Magnetic Resonance Imaging Clinics of North America, vol. 17, no. 2, pp. 263-275, 2009.

[20] J.-F. Budzik, V. Balbi, S. Verclytte, V. Pansini, V. le Thuc, and A. Cotten, "Diffusion tensor imaging in musculoskeletal disorders," Radiographics, vol. 34, no. 3, pp. E56-E72, 2014.
[21] O. Dietrich, A. Biffar, A. Baur-Melnyk, and M. F. Reiser, "Technical aspects of MR diffusion imaging of the body," European Journal of Radiology, vol. 76, no. 3, pp. 314-322, 2010.

[22] D. Jaramillo, "Whole-body MR imaging, bone diffusion imaging: how and why?" Pediatric Radiology, vol. 40, no. 6, pp. 978$984,2010$.

[23] H. K. Kim, D. M. Lindquist, S. D. Serai et al., "Magnetic resonance imaging of pediatric muscular disorders. Recent advances and clinical applications," Radiologic Clinics of North America, vol. 51, no. 4, pp. 721-742, 2013.

[24] A. Baur-Melnyk and H. Boehm, "Osteoporosis," in Screening and Preventive Diagnosis with Radiological Imaging, M. F. Reiser, G. van Kaick, C. Fink, and S. O. Schoenberg, Eds., pp. 249-260, Springer, Berlin, Germany, 2008.

[25] H. K. Genant and Y. Jiang, "Advanced imaging assessment of bone quality," Annals of the New York Academy of Sciences, vol. 1068, no. 1, pp. 410-428, 2006.

[26] World Health Organization, "Assessment of fracture risk and its application to screening for postmenopausal osteoporosis: report of a WHO study group," World Health Organization Technical Report Series vol. 843, World Health Organization, Geneva, Switzerland, 1994.

[27] J. A. Kanis, "Diagnosis of osteoporosis and assessment of fracture risk," The Lancet, vol. 359, no. 9321, pp. 1929-1936, 2002.

[28] B. Cortet and X. Marchandise, "Bone microarchitecture and mechanical resistance," Joint Bone Spine, vol. 68, no. 4, pp. 297305, 2001.

[29] J. F. Griffith and H. K. Genant, "Bone mass and architecture determination: state of the art," Best Practice and Research: Clinical Endocrinology and Metabolism, vol. 22, no. 5, pp. 737764, 2008.

[30] P. McDonnell, P. E. McHugh, and D. O'Mahoney, "Vertebral osteoporosis and trabecular bone quality," Annals of Biomedical Engineering, vol. 35, no. 2, pp. 170-189, 2007.

[31] J. S. Bauer and T. M. Link, "Advances in osteoporosis imaging," European Journal of Radiology, vol. 71, no. 3, pp. 440-449, 2009.

[32] NIH Consensum Development Panel on Osteoporosis Prevention, "Osteoporosis prevention, diagnosis, and therapy," The Journal of the American Medical Association, vol. 285, no. 6, pp. 785-795, 2001.

[33] F. W. Wehrli, J. C. Ford, M. Attie, H. Y. Kressel, and F. S. Kaplan, "Trabecular structure: preliminary application of MR interferometry," Radiology, vol. 179, no. 3, pp. 615-621, 1991.

[34] F. W. Wehrli, J. A. Hopkins, S. N. Hwang, H. K. Song, P. J. Snyder, and J. G. Haddad, "Cross-sectional study of osteopenia with quantitative MR imaging and bone densitometry," Radiology, vol. 217, no. 2, pp. 527-538, 2000.

[35] T. M. Link, S. Majumdar, P. Augat et al., "Proximal femur: assessment for osteoporosis with $\mathrm{T} 2 *$ decay characteristics at MR imaging," Radiology, vol. 209, no. 2, pp. 531-536, 1998.

[36] P. K. Saha, B. R. Gomberg, and F. W. Wehrli, "Three-dimensional digital topological characterization of cancellous bone architecture," International Journal of Imaging Systems and Technology, vol. 11, no. 1, pp. 81-90, 2000.

[37] R. Krug, S. Banerjee, E. T. Han, D. C. Newitt, T. M. Link, and S. Majumdar, "Feasibility of in vivo structural analysis of highresolution magnetic resonance images of the proximal femur," Osteoporosis International, vol. 16, no. 11, pp. 1307-1314, 2005.

[38] S. Majumdar, D. Thomasson, A. Shimakawa, and H. K. Genant, "Quantitation of the susceptibility difference between trabecular bone and bone marrow: experimental studies," Magnetic Resonance in Medicine, vol. 22, no. 1, pp. 111-127, 1991. 
[39] S. N. Hwang and F. W. Wehrli, "The calculation of the susceptibility-induced magnetic field from 3D NMR images with applications to trabecular bone," Journal of Magnetic Resonance, Series B, vol. 109, no. 2, pp. 126-145, 1995.

[40] S. de Santis, M. Rebuzzi, G. Di Pietro, F. Fasano, B. Maraviglia, and S. Capuani, "In vitro and in vivo MR evaluation of internal gradient to assess trabecular bone density," Physics in Medicine and Biology, vol. 55, no. 19, pp. 5767-5785, 2010.

[41] F. W. Wehrli, "Magnetic resonance of calcified tissues," Journal of Magnetic Resonance, vol. 229, pp. 35-48, 2013.

[42] T. H. Berquist, MRI of the Musculoskeletal System, Lippincott Williams \& Wilkins, 5th edition, 2006.

[43] S. Capuani, "Water diffusion in cancellous bone," Microporous and Mesoporous Materials, vol. 178, pp. 34-38, 2013.

[44] D. Schellinger, C. S. Lin, J. Lim, H. G. Hatipoglu, J. C. Pezzullo, and A. J. Singer, "Bone marrow fat and bone mineral density on proton MR spectroscopy and dual-energy X-ray absorptiometry: their ratio as a new indicator of bone weakening," The American Journal of Roentgenology, vol. 183, no. 6, pp. 1761-1765, 2004.

[45] D. K. W. Yeung, J. F. Griffith, G. E. Antonio, F. K. H. Lee, J. Woo, and P. C. Leung, "Osteoporosis is associated with increased marrow fat content and decreased marrow fat unsaturation: a proton MR spectroscopy study," Journal of Magnetic Resonance Imaging, vol. 22, no. 2, pp. 279-285, 2005.

[46] J. F. Griffith, D. K. W. Yeung, G. E. Antonio et al., "Vertebral bone mineral density, marrow perfusion, and fat content in healthy men and men with osteoporosis: dynamic contrast-enhanced MR imaging and MR spectroscopy," Radiology, vol. 236, no. 3, pp. 945-951, 2005.

[47] M. C. Walsh, G. R. Hunter, and M. B. Livingstone, "Sarcopenia in premenopausal and postmenopausal women with osteopenia, osteoporosis and normal bone mineral density," Osteoporosis International, vol. 17, no. 1, pp. 61-67, 2006.

[48] U. Tarantino, J. Baldi, M. Celi et al., "Osteoporosis and sarcopenia: the connections," Aging Clinical and Experimental Research, vol. 25, no. 1, pp. S93-S95, 2013.

[49] G. P. Liney, C. P. Bernard, D. J. Manton, L. W. Turnbull, and C. M. Langton, "Age, gender, and skeletal variation in bone marrow composition: a preliminary study at 3.0 Tesla," Journal of Magnetic Resonance Imaging, vol. 26, no. 3, pp. 787-793, 2007.

[50] F. H. Netter, R. T. Woodburne, E. S. Crelin, and F. Kaplan, Musculoskeletal System, Part 1: Anatomy, Physiology, and Metabolic Disorders, vol. 8 of Netter Collection of Medical Illustrations, Ciba Pharmaceutical, 1987.

[51] J. Ren, I. Dimitrov, A. D. Sherry, and C. R. Malloy, "Composition of adipose tissue and marrow fat in humans by ${ }^{1} \mathrm{H}$ NMR at 7 Tesla," Journal of Lipid Research, vol. 49, no. 9, pp. 2055-2062, 2008.

[52] S. A. Athavale, S. D. Joshi, and S. S. Joshi, "Internal architecture of calcaneus: correlations with mechanics and pathoanatomy of calcaneal fractures," Surgical and Radiologic Anatomy, vol. 32, no. 2, pp. 115-122, 2010.

[53] S. Capuani, E. Piccirilli, G. di Pietro, M. Celi, and U. Tarantino, "Microstructural differences between osteoporotic and osteoarthritic femoral cancellous bone: an in vitro magnetic resonance micro-imaging investigation," Aging Clinical and Experimental Research, vol. 25, no. 1, pp. S51-S54, 2013.

[54] C. J. Rosen and M. L. Bouxsein, "Mechanisms of disease: is osteoporosis the obesity of bone?" Nature Clinical Practice Rheumatology, vol. 2, no. 1, pp. 35-43, 2006.
[55] M. Rebuzzi, V. Vinicola, F. Taggi, U. Sabatini, F. W. Wehrli, and S. Capuani, "Potential diagnostic role of the MRI-derived internal magnetic field gradient in calcaneus cancellous bone for evaluating postmenopausal osteoporosis at 3T,' Bone, vol. 57, no. 1, pp. 155-163, 2013.

[56] W. S. Price, "Pulsed-field gradient nuclear magnetic resonance as a tool for studying translational diffusion. Part 1. Basic theory," Concepts in Magnetic Resonance, vol. 9, no. 5, pp. 299335, 1997.

[57] E. O. Stejskal and J. E. Tanner, "Spin diffusion measurements: spin echoes in the presence of a time-dependent field gradient," The Journal of Chemical Physics, vol. 42, no. 1, pp. 288-292, 1965.

[58] U. Sinha, S. Sinha, J. A. Hodgson, and R. V. Edgerton, "Human soleus muscle architecture at different ankle joint angles from magnetic resonance diffusion tensor imaging," Journal of Applied Physiology, vol. 110, no. 3, pp. 807-819, 2011.

[59] J. F. Budzik, V. Le Thuc, X. Demondion, M. Morel, D. Chechin, and A. Cotten, "In vivo MR tractography of thigh muscles using diffusion imaging: initial results," European Radiology, vol. 17, no. 12, pp. 3079-3085, 2007.

[60] J. E. Tanner, "Use of stimulated echo in NMR-diffusion studies," The Journal of Chemical Physics, vol. 52, no. 5, pp. 2523-2526, 1970.

[61] D. K. Jones and P. J. Basser, "Squashing peanuts and smashing pumpkins': how noise distorts diffusion-weighted MR data," Magnetic Resonance in Medicine, vol. 52, no. 5, pp. 979-993, 2004.

[62] M. E. Bastim, P. A. Armitage, and I. Marshall, "A theoretical SNR: non monotonic behaviors of tensor contrasts," Magnetic Resonance Imaging, vol. 16, pp. 773-785, 1998.

[63] B. A. Landman, J. A. D. Farrell, H. Huang, J. L. Prince, and S. Mori, "Diffusion tensor imaging at low SNR: nonmonotonic behaviors of tensor contrasts," Magnetic Resonance Imaging, vol. 26, no. 6, pp. 790-800, 2008.

[64] G. Manenti, S. Capuani, E. Fanucci et al., "Diffusion tensor imaging and magnetic resonance spectroscopy assessment of cancellous bone quality in femoral neck of healthy, osteopenic and osteoporotic subjects at 3T: preliminary experience," Bone, vol. 55, no. 1, pp. 7-15, 2013.

[65] B. M. Damon, Z. Ding, A. W. Anderson, A. S. Freyer, and J. C. Gore, "Validation of diffusion tensor MRI-based muscle fiber tracking," Magnetic Resonance in Medicine, vol. 48, no. 1, pp. 97104, 2002.

[66] U. Sinha and L. Yao, "In vivo diffusion tensor imaging of human calf muscle," Journal of Magnetic Resonance Imaging, vol. 15, no. 1, pp. 87-95, 2002.

[67] A. van Doorn, P. H. M. Bovendeerd, K. Nicolay, M. R. Drost, and J. D. Janssen, "Determination of muscle fibre orientation using diffusioni-weighted MRI," European Journal of Morphology, vol. 34, no. 1, pp. 5-10, 1996.

[68] C. Rossi, A. Boss, G. Steidle et al., "Water diffusion anisotropy in white and gray matter of the human spinal cord," Journal of Magnetic Resonance Imaging, vol. 27, no. 3, pp. 476-482, 2008.

[69] F. Schick, "Bone marrow NMR in vivo," Progress in Nuclear Magnetic Resonance Spectroscopy, vol. 29, no. 3-4, pp. 169-227, 1996.

[70] Y. Ueda, T. Miyati, N. Ohno et al., "Apparent diffusion coefficient and fractional anisotropy in the vertebral bone marrow," Journal of Magnetic Resonance Imaging, vol. 31, no. 3, pp. 632-635, 2010.

[71] F. W. Wehrli, L. Hilaire, M. Fernández-seara et al., "Quantitative magnetic resonance imaging in the calcaneus and femur 
of women with varying degrees of osteopenia and vertebral deformity status," Journal of Bone and Mineral Research, vol. 17, no. 12, pp. 2265-2273, 2002.

[72] J. F. Griffith, D. K. W. Yeung, G. E. Antonio et al., "Vertebral marrow fat content and diffusion and perfusion indexes in women with varying bone density: MR evaluation," Radiology, vol. 241, no. 3, pp. 831-838, 2006.

[73] D. K. W. Yeung, S. Y. S. Wong, J. F. Griffith, and E. M. C. Lau, "Bone marrow diffusion in osteoporosis: evaluation with quantitative MR diffusion imaging," Journal of Magnetic Resonance Imaging, vol. 19, no. 2, pp. 222-228, 2004.

[74] S. Capuani, C. Rossi, M. Alesiani, and B. Maraviglia, "Diffusion tensor imaging to study anisotropy in a particular porous system: the trabecular bone network," Solid State Nuclear Magnetic Resonance, vol. 28, no. 2-4, pp. 266-272, 2005.

[75] G. Di Pietro, M. Palombo, and S. Capuani, "Internal magnetic field gradients in heterogeneous porous systems: comparison between Spin-Echo and Diffusion Decay Internal Field (DDIF) method," Applied Magnetic Resonance, vol. 45, no. 8, pp. 771784, 2014

[76] J. Mitchell, T. C. Chandrasekera, M. L. Johns, L. F. Gladden, and E. J. Fordham, "Nuclear magnetic resonance relaxation and diffusion in the presence of internal gradients: the effect of magnetic field strength," Physical Review E: Statistical, Nonlinear, and Soft Matter Physics, vol. 81, no. 2, Article ID 026101, 2010.

[77] E. E. Sigmund, H. Cho, and Y.-Q. Song, "High-resolution MRI of internal field diffusion-weighting in trabecular bone," $N M R$ in Biomedicine, vol. 22, no. 4, pp. 436-448, 2009.

[78] J. Zhong, R. P. Kennan, and J. C. Gore, "Effects of susceptibility variations on NMR measurements of diffusion," Journal of Magnetic Resonance, vol. 95, no. 2, pp. 267-280, 1991.

[79] C. Rossi, A. Boss, P. Martirosian et al., "Influence of steady background gradients on the accuracy of molecular diffusion anisotropy measurements," Magnetic Resonance Imaging, vol. 26, no. 9, pp. 1250-1258, 2008. 


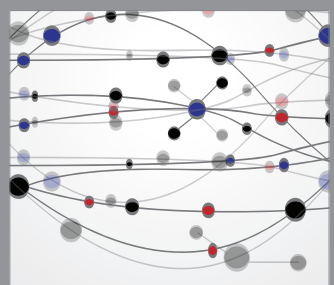

The Scientific World Journal
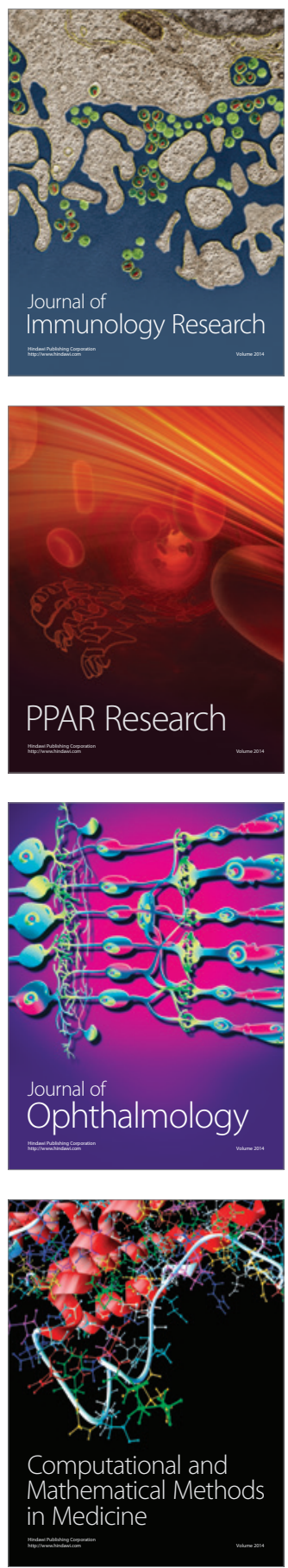

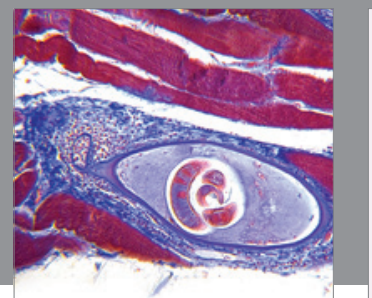

Gastroenterology

Research and Practice
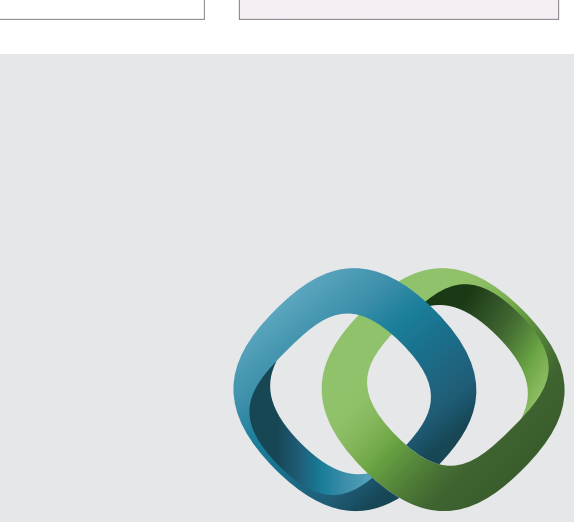

\section{Hindawi}

Submit your manuscripts at

http://www.hindawi.com
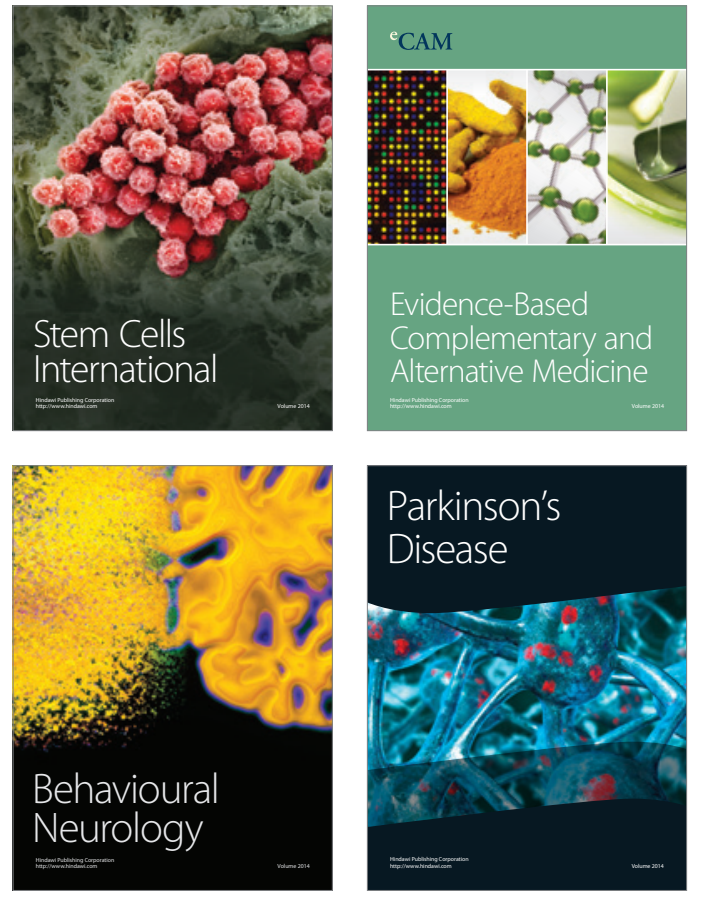
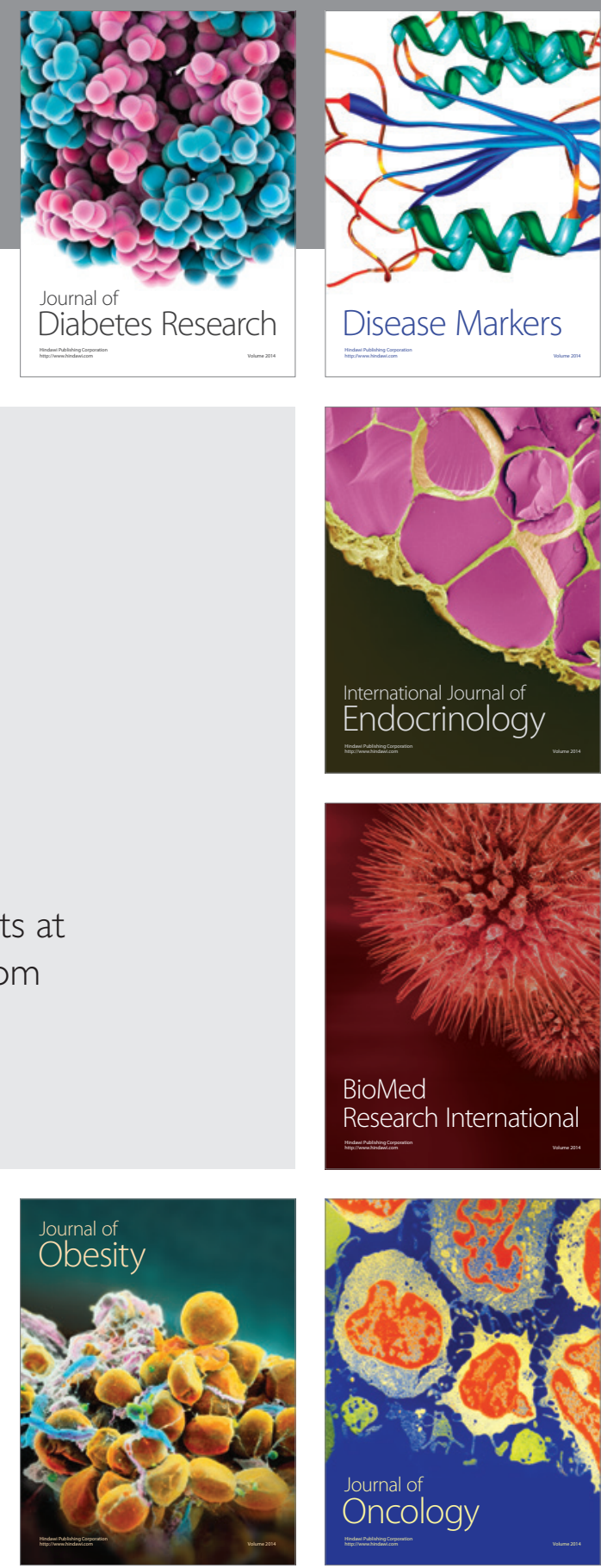

Disease Markers
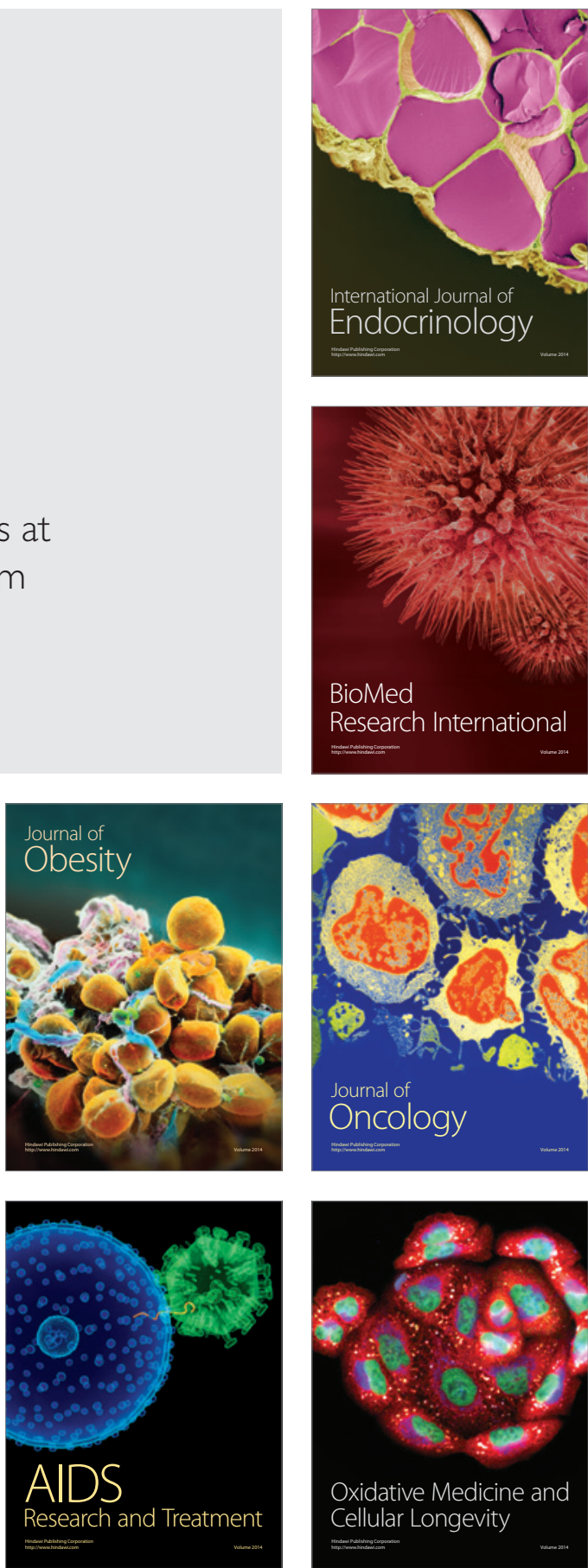